\title{
EVOLUÇÃO DA INFECÇÃO POR NEISSERIA GONORRHOEAE NUMA POPULAÇÃO DA CONSULTA DE DST DO CENTRO DE SAÚDE DA LAPA DE 2007 A 2011
}

\author{
Hugo Barreiros', Jacinta Azevedo², Irene Santo² \\ 'Interno do Internato Complementar de Dermatologia e Venereologia / Resident of Dermatology and Venereology, \\ Serviço de Dermatologia e Venereologia / Department of Dermatology and Venereology, Hospital Garcia de Orta, \\ Almada, Portugal \\ ${ }^{2}$ Assistente Hospitalar de Dermatovenereologia, Chefe de Serviço de Medicina Geral e Familiar / Consultant of \\ Dermatology and Venereology, Consultant Chief of General and Family Practitioner, Consulta de Doenças Sexualmente \\ Transmissiveis / Outpatient Clinic of STDs, Centro de Saúde da Lapa, Lisboa, Portugal
}

RESUMO - Introdução: No final da década de 70 observou-se, na grande maioria dos países da Europa Ocidental, um decréscimo da incidência de gonorreia. Em meados dos anos 90, assistiu-se a um importante ressurgimento da doença nalguns países e, mais recentemente, a incidência tem aumentado nalgumas populações específicas, de maior risco. Material e métodos: Os autores analisam, retrospectivamente, os processos clínicos da população observada na consulta de Doenças Sexualmente Transmissíveis (DST) do Centro de Saúde da Lapa (C S Lapa), entre Janeiro de 2007 e Dezembro de 2011 (5 anos). Procede-se à caracterização epidemiológica, determinação da frequência da infecção por Neisseria gonorrhoeae $(\mathrm{Ng})$, localização anatómica e avaliação dos factores sociodemográficos associados. Resultados: Durante o período em análise recorreram à consulta 9068 indivíduos e foram diagnosticados 386 (4.3\%) casos de gonorreia (335 homens e 51 mulheres, com uma média de idades de 30 anos). Em 2007 ocorreram 66 casos de gonorreia, 57 em 2008, 106 em 2009, 67 em 2010 e 90 em 2011. A localização uretral foi a mais frequente, com 312 casos, seguindo-se a infecção do colo do útero em 51 mulheres e do recto em 30 homens. Verificaram-se apenas 2 casos de infecção gonocócica da orofaringe. As co-infecções foram frequentes, salientando-se 80 casos com Chlamydia trachomatis e 50 com infecção VIH. Conclusões: $O$ número de infecções gonocócicas diagnosticadas na consulta de DST do CS da Lapa tem aumentado gradualmente ao longo da última década, com um pico de incidência em 2009. Importa, por isso, considerar a infecção por $N$. gonorrhoeae como um problema actual, longe da erradicação e que tem colocado novos desafios a nível do tratamento.

PALAVRAS-CHAVE - Neisseria gonorrhoeae; Infecções sexualmente transmissíveis; Factores sociodemográficos.

\section{GONOCOCCAL INFECTION IN A SEXUALLY TRANSMITTED INFECTION CLINIC. A RETROSPECTIVE STUDY OF FIVE YEARS: 2007-2011}

ABSTRACT - Introduction: In the late 70's gonococcal infection decreased in most Western European Countries. In the 90's there has been an important increase in the incidence of the disease, mostly in some higher risk groups. Material and methods: This is a retrospective study based in the population attending the STD Clinic of Centro de Saúde da Lapa, between January 2007 and December 2011 (5 years). We assessed epidemiological data, the prevalence of Neisseria gonorrhoeae infection and associated sociodemographic factors. Results: During the study period a total of 9068 patients attended the Clinic. Gonococcal infection was diagnosed in 386 (4.3\%) patients (335 men and 51 women, median age of 30). In 2007 there were 66 cases of gonorrhoea, 57 cases in 2008, 106 cases in 2009, 67 cases in 2010 and 90 cases in 2011 . The most frequent localization of infection was the urethra with 312 cases, followed by uterine cervix in 51 women and rectus in 30 men, with only 2 infections in the oropharynge. Co-infection was found with 
Chlamydia trachomatis in 80 cases and HIV in 50 patients. Conclusions: Gonococcal infections diagnosed in the STD Clinic of Centro de Saúde da Lapa have gradually increased in the last decade, with most cases diagnosed in 2009. N. gonorrhoeae infection is a current problem, far from being eradicated and with new challenges regarding treatment.

KEY-WORDS - Neisseria gonorrhoeae; Sexually transmitted diseases.

\begin{abstract}
Conflitos de interesse: Os autores declaram não possuir conflitos de interesse.
No conflicts of interest.

Suporte financeiro: $O$ presente trabalho não foi suportado por nenhum subsídio ou bolsa.

No sponsorship or scholarship granted.

Responsabilidades éticas e protecção de pessoas e animais / Ethical responsibilities and human and animal protections: Os autores declaram que para esta investigação não se realizaram experiências em seres humanos e/ou animais.

The authors declare that no experiences with humans or animals were undertaken for the present investigation. Confidencialidade dos dados / Confidentiality agreement: Os autores declaram ter seguido os protocolos éticos acerca da publicação dos dados de doentes e que todos os doentes incluídos no estudo receberam informações suficientes e deram o seu consentimento informado por escrito para participar nesse estudo. The authors declare that all ethical procedures related to the publication of patient's data were followed and that all patients included in the study were completely informed and gave a written informed consent to participate in the study.

Direito à privacidade e consentimento escrito / Privacy policy and informed consent: Os autores declaram que não aparecem dados de doentes neste artigo.

The authors declare that no patients' data are shown in this article.
\end{abstract}

Recebido/Received - Setembro/September 2012; Aceite/Accepted - Novembro/November 2012

Por decisão dos autores, este artigo não foi redigido de acordo com os termos do novo Acordo Ortográfico.

\title{
Correspondêncie \\ Dr. Hugo Barreiros
}

Serviço de Dermatologia e Venereologia

Hospital Garcia de Orta

Av. Torrado Silva

2805-267 Almada, Portugal

Tel.: + 351212727100

Fax: + 351212957004

E-mail: hbarreiros@gmail.com

\section{INTRODUÇÃO}

A infecção gonocócica é causada pela bactéria Neisseria gonorrhoeae $(\mathrm{Ng})$. Este agente foi isolado pela primeira vez em 1879 pelo dermatologista alemão Albert Ludwig Sigesmund Neisser' que, não tendo conseguido colocação como internista, acabou, por mero acaso, por trabalhar como assistente de Oskar Simon, conceituado dermatologista da época ${ }^{2}$. Albert Neisser dedica-se de início e quase exclusivamente às doenças venéreas e à lepra (ainda não conhecida como Doença de Hansen) ${ }^{3}$ e, aos 24 anos, identifica pela primeira vez o microorganismo responsável pela infecção gonocócica. Causa frequente de uretrites e cervicites, infecções que tiveram ao longo do tempo várias denominações, umas científicas outras mais populares, entre elas: uretrite gonocócica; gonorreia; blenorragia; blenorreia; esquentamento; gota matinal; gota militar; pingadeira; fogagem. 
A Neisseria gonorrhoeae pertence à família das Neisseriaceae, tratando-se de um diplococo gram-negativo não flagelado e de localização intracelular nos polimorfonucleares nas fases iniciais da doença gonocócica. Este patogéneo tem uma apetência especial pela mucosa uretral, facto que permite explicar o número elevado de casos referidos nesta localização. Estima-se que a incidência mundial ronde os 88 milhões de casos anuais, atingindo particularmente os países subdesenvolvidos ${ }^{4}$. Nos Estados Unidos são cerca de 800.000 casos/ano com um custo estimado de 1 bilião de dólares ${ }^{5}$.

No final da década de 70 observou-se, na grande maioria dos países da Europa Ocidental, um decréscimo da incidência de gonorreia. Em meados dos anos 90, porém, verificou-se um importante recrudescimento da doença nalguns países e, mais recentemente, um aumento da incidência nalgumas populações específicas, de maior risco para aquisição de infecções sexualmente transmissíveis (IST) ${ }^{6}$. A infecção é muito mais frequente nos homens do que nas mulheres, que são geralmente assintomáticas, nos jovens, ocorrendo $75 \%$ dos casos em indivíduos na faixa etária dos 15 aos 29 anos, e nos estratos socioeconómicos mais baixos. Na última década, o aumento da incidência foi mais acentuada nos homens que praticam sexo com homens $(\mathrm{HSH})^{7}$.

A transmissão da infecção ocorre por relação sexual não protegida, com a possibilidade de infecção via genital-genital, genital-ororectal ou oro-genital. Outra possibilidade de transmissão é por via vertical, da mãe para $\circ$ filho durante $\circ$ parto ${ }^{8}$. A bactéria coloniza as mucosas (genito-urinárias, recto, faringe, conjuntivas). Após a inoculação pode ocorrer uma disseminação ascendente, responsável por complicações graves como a doença inflamatória pélvica (DIP) 9 ou a epidídimo-orquite. Raramente ocorre uma disseminação por via hematogénea ${ }^{8}$. Além das complicações já referidas, a infecção gonocócica pode provocar gravidez ectópica e infertilidade (tanto feminina como masculina), e facilita a transmissão do vírus $\mathrm{VIH}$ (vírus da imunodeficiência humana) ${ }^{10}$.

\section{OBJECTIVOS}

Os autores analisam, retrospectivamente, a prevalência da infecção gonocócica na população observada na consulta de DST (Doenças Sexualmente Transmissíveis) do CS Lapa (Centro de Saúde da Lapa), entre Janeiro de 2007 e Dezembro de 2011. Avaliam-se os factores sociodemográficos associados à infecção gonocócica, assim como a presença de co-infecções na altura do diagnóstico. Por último, comparam-se os resultados com estudo anterior ${ }^{11}$ efectuado na mesma Consulta.

\section{MATERIAIS E MÉTODOS}

A pesquisa de Neisseria gonorrhoeae é efectuada sistematicamente nos doentes com sintomas, nos parceiros sexuais dos doentes com gonorreia e nas muIheres que vêm pela primeira vez à consulta. Os testes laboratoriais microbiológicos são realizados no Instituto Nacional de Saúde Dr. Ricardo Jorge, a partir das amostras de exsudado cervical, uretral, anal e/ou orofaringe para cultura em meio de Thayer-Martin enriquecido (conservação com placas mantidas a $37^{\circ} \mathrm{C}$ em anaerobiose). Nos homens assintomáticos e em algumas mulheres a identificação de Neisseria gonorrhoeae é feita em amostras de urina por métodos de PCR (Polymerase Chain Reaction). São considerados critérios para definição de caso de infecção gonocócica a presença de diplococos intracelulares (em polimorfonucleares) no exame microscópico a fresco de esfregaço uretral corado pelo azul de metileno e/ou gonocultura positiva, ou PCR-Ng positiva.

$\mathrm{Na}$ análise retrospectiva dos processos, foram analisadas as variáveis sociodemográficas incluindo: género; idade; origem; escolaridade; orientação sexual; número de parceiros sexuais nos últimos 6 meses. Avaliou-se ainda a presença de outras IST na altura do diagnóstico e história de IST prévias.

\section{RESULTADOS}

Durante o período compreendido entre Janeiro de 2007 e Dezembro de 2011, recorreram à consulta 9068 indivíduos e foram diagnosticados 386 casos de gonorreia (335 homens - $86 \%$ - e 51 mulheres - 14\%, com uma média de idades de 30 anos). A Fig. 1 ilustra o total de consultas e a sua distribuição por género em cada ano do período de estudo. A Fig. 2 exibe o número de diagnósticos de gonorreia por ano e por género. De salientar o pico de incidência de infecções gonocócicas que se verificou em 2009. Em 2010 há um decréscimo de diagnósticos para valores semelhantes a 2007 e 2008, seguindo-se de um novo recrudescimento em 2011. A localização mais frequente de gonorreia (Fig. 3) foi a uretra com 312 infecções, 


\section{GEIDST}

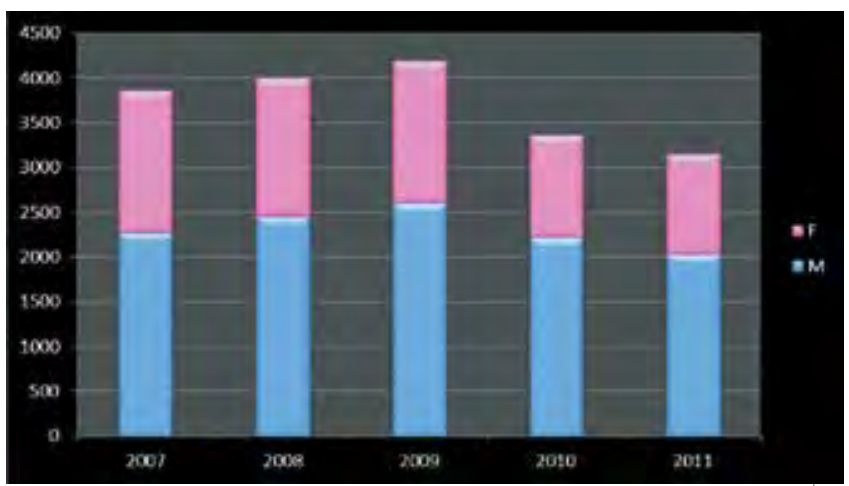

Fig. 1 - Total de consultas de 2007 - 2011 (distribuição por género).

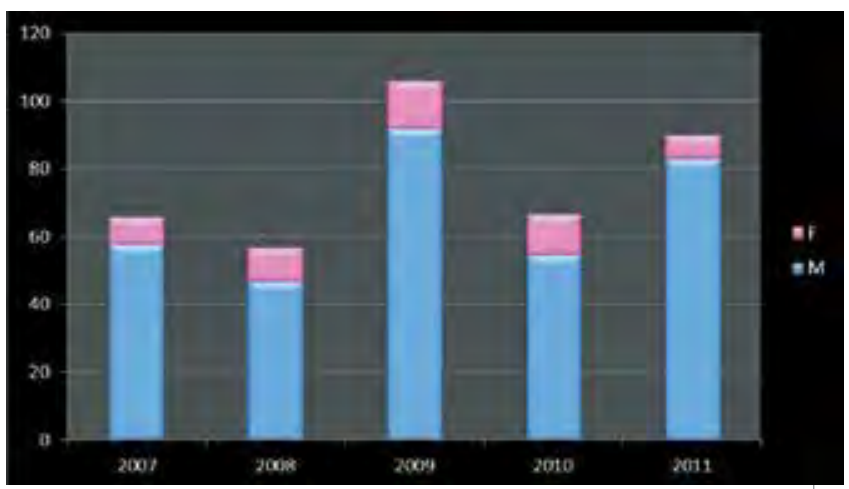

Fig. 2 - Diagnósticos de gonorreia 2007 - 2011 (distribuição por género).

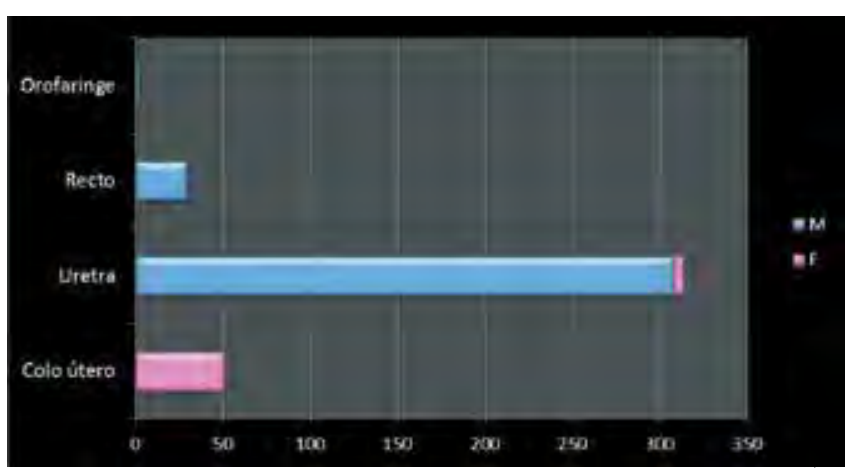

Fig. 3 - Localização das infecções de gonorreia.

seguida do colo do útero em 51 mulheres e recto em 30 homens, com apenas 2 infecções da orofaringe. Os grupos etários de infecção gonocócica são os inicialmente esperados para uma consulta de DST, dirigida a uma população sexualmente activa e as idades variaram entre os 14 e os 69 anos. Verificaram-se 49 casos para o grupo com idade inferior ou igual a 20 anos; 190 casos no grupo entre os $21-30$ anos; 102 casos entre os 31-40 anos; 30 casos entre os 41-50; 11 casos entre os 51-60; e 5 casos em indivíduos com mais de 60 anos.

A Tabela I caracteriza os 386 doentes com diagnóstico de gonorreia quanto à origem, escolaridade, orientação sexual e número de parceiros nos últimos 6 meses, Ao analisar a origem dos doentes com infecção gonocócica destaca-se o facto de 158 (41\%) não serem naturais de Portugal. A grande percentagem de imigrantes na consulta é não só um reflexo das mudanças verificadas no nosso país nas últimas décadas mas também resultado do facto de se tratar de uma consulta aberta, com acesso fácil aos imigrantes, incluindo os indocumentados. 292 doentes (76\%) concluíram o ensino secundário ou superior, uma percentagem importante dos infectados $(75 \%)$ referia mais do que um parceiro sexual nos últimos 6 meses. 48\% (185) das infecções ocorreram em homens que praticam sexo com homens (HSH).

Tabela 1 - Caracterização dos indivíduos com infecção gonocócica

\begin{tabular}{|c|c|c|c|c|}
\hline & & $\mathbf{M}$ & $\mathbf{F}$ & TOTAL \\
\hline \multirow{5}{*}{ País de Origem } & Portugal & 189 & 39 & 228 \\
\hline & Europa & 19 & 1 & 20 \\
\hline & PALOP & 54 & 8 & 62 \\
\hline & Brasil & 64 & 3 & 67 \\
\hline & Outros & 9 & 0 & 9 \\
\hline \multirow{5}{*}{ Escolaridade } & Analfabeto & 0 & 0 & 0 \\
\hline & Primário & 68 & 24 & 92 \\
\hline & Secundário & 145 & 18 & 163 \\
\hline & Superior & 120 & 9 & 129 \\
\hline & Desconhecido & 2 & 0 & 2 \\
\hline \multirow{3}{*}{$\begin{array}{l}\text { Orientação } \\
\text { Sexual }\end{array}$} & Homo & 127 & 0 & 127 \\
\hline & Hetero & 150 & 50 & 200 \\
\hline & Bissexual & 58 & 1 & 59 \\
\hline \multirow{4}{*}{$\begin{array}{c}\mathrm{N}^{\circ} \text { de parceiros } \\
(6 \mathrm{M})\end{array}$} & 0 & 3 & 0 & 3 \\
\hline & 1 & 56 & 36 & 92 \\
\hline & $2-4$ & 195 & 12 & 207 \\
\hline & $\geq 5$ & 81 & 3 & 84 \\
\hline
\end{tabular}


A Tabela 2 ilustra as IST diagnosticadas previamente ou concomitantes com a infecção gonocócica. Registaram-se 142 co-infecções, salientando-se 80 casos de infecção por Chlamydia trachomatis, 18 de sífilis recente el6 de infecção VIH "de novo" (13 dos quais com teste $\mathrm{VIH}$ anterior negativo). 35 casos de gonorreia ocorreram em doentes com infecção $\mathrm{VIH}$ conhecida.

\section{Tabela 2 - Antecedentes / co-infecção de DST em doentes com infecção gonocócica}

\begin{tabular}{|c|c|c|c|c|}
\hline & & $\mathbf{M}$ & $\mathbf{F}$ & TOTAL \\
\hline \multirow{6}{*}{$\begin{array}{c}\text { Antecedentes } \\
\text { de DST }\end{array}$} & CT & 34 & 3 & 37 \\
\hline & Gonorreia & 51 & 4 & 55 \\
\hline & Sífilis & 31 & 2 & 33 \\
\hline & Condilomas & 12 & 1 & 13 \\
\hline & Herpes & 11 & 0 & 11 \\
\hline & HIV & 34 & 1 & 35 \\
\hline \multirow{9}{*}{$\begin{array}{l}\text { Co-infecção } \\
\text { (outras DST) }\end{array}$} & $\mathrm{CT}$ & 63 & 17 & 80 \\
\hline & Sífilis recente & 18 & 0 & 18 \\
\hline & Sífilis ind. & 6 & 0 & 6 \\
\hline & Condilomas & 8 & 7 & 15 \\
\hline & Herpes & 5 & 0 & 5 \\
\hline & Tricomoníase & 0 & 2 & 2 \\
\hline & HIV & 15 & 1 & 16 \\
\hline & Candidíase VV & 0 & 4 & 4 \\
\hline & Vaginose & 0 & 1 & 1 \\
\hline
\end{tabular}

\section{DISCUSSÃO E CONCLUSÕES}

Os dados deste estudo não são representativos da população portuguesa, nem mesmo da cidade de Lisboa, dadas as características dos utentes da Consulta (população jovem, elevado nível educacional, grande percentagem de $\mathrm{HSH}$ ).

Os nossos dados indicam uma prevalência elevada de infecção gonocócica (4.3\%), particularmente em homens jovens. A média de idades dos doentes com gonorreia foi de 30 anos, $86 \%$ eram do sexo masculino, com um ratio homem-mulher 6.6:1. A infecção da uretra foi a mais frequente, correspondendo a $81 \%$ dos casos, o que está de acordo com a grande afinidade da bactéria para a mucosa uretral.
Verificou-se um número muito importante de casos em grupos específicos, nomeadamente os homens que praticam sexo com homens, com $48 \%$ dos casos e os indivíduos com mais de um parceiro sexual, reunindo $75 \%$ dos casos. Muito se tem debatido acerca das razões do recrudescimento da infecção nos últimos anos, sendo o aumento entre grupos populacionais específicos um facto globalmente aceite. Salienta-se o número elevado de co-infecção com Chlamydia trachomatis (80 casos - 21\%), o que está de acordo com a literatura, pelo que é recomendado, nos doentes com uretrite e/ou cervicite, um regime terapêutico eficaz simultaneamente contra a gonorreia e a infecção por clamídia ${ }^{12}$.

A importância das IST como factor de risco para a aquisição e transmissão de infecção pelo $\mathrm{VIH}$ tem sido reiterada a nível mundial No nosso estudo 35 doentes com infecção gonocócica eram seropositivos para - $\mathrm{VIH}$ e, em 16 casos foi diagnosticada a infecção $\mathrm{VIH}$ (4\%). Assim, em 51 doentes (13\%) observou-se a associação VIH - gonorreia.

Compararam-se os resultados deste estudo com os de um estudo anterior ${ }^{11}$ efectuado na mesma Consulta, no período compreendido entre 1998 e 2006 . O nosso estudo vem dar sequência aos resultados nos cinco anos seguintes (2007 a 2011). A Fig. 4 junta os resultados de ambos os estudos ilustrando a evolução dos diagnósticos de gonorreia desde 1998 até 2011. De notar que o recurso a técnicas de pesquisa de Neisseria gonorrhoeae por métodos moleculares pode ter influenciado o número de diagnósticos nos últimos anos. Efectivamente, a pesquisa por estes métodos iniciou-se na Consulta em Julho de 2005, o que veio aumentar a sensibilidade permitindo também o diagnóstico de doentes assintomáticos.

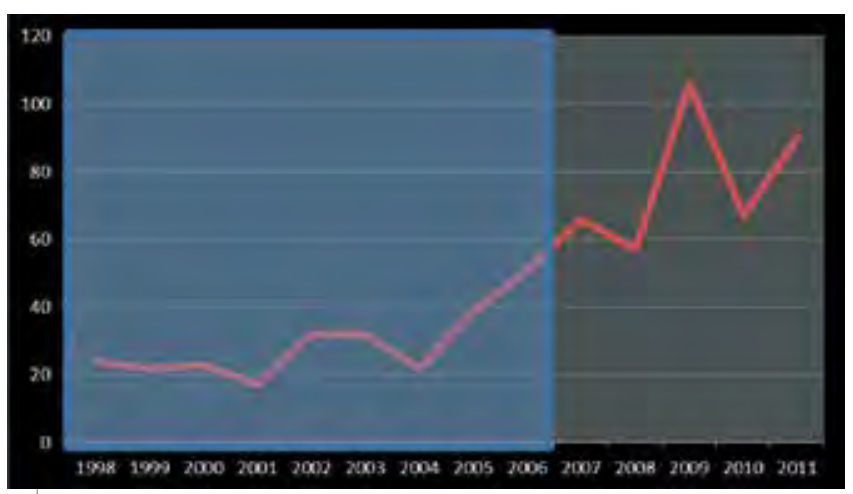

Fig 4 - Número de diagnósticos de gonorreia na Consulta de DST do CS Lapa de 1998 a 2011. 
Actualmente não é possível um conhecimento fundamentado da incidência de infecção gonocócica em Portugal. Por um lado, se o recurso limitado a meios laboratoriais diferenciados não permite na maioria dos casos o diagnóstico etiológico, por outro, mesmo quando o diagnóstico é feito, o número de casos que são notificados é muito inferior ao número real.

O número de infecções gonocócicas diagnosticadas na consulta de DST do CS da Lapa tem vindo gradualmente a aumentar ao longo da última década, com pico de incidência em 2009. Importa, por isso, considerar a infecção por $N$. gonorrhoeae como um problema actual, longe da erradicação e que tem colocado novos desafios a nível do tratamento ${ }^{13-16}$.

\section{BIBLIOGRAFIA}

1. Neisser A. Ueber cine der Gonorrhoe eigentumliche Micrococcusform. Zentralblati flur die Medizinischen Wissenschaften. 1879; 17:497-500.

2. Oriel JD. Eminent venereologists. 1. Albert Neisser. Genitourin Med. 1989; 65:235-8.

3. Neisser A. Zur Aetiologie der Lepra. Breslauer Artze Zeitschrift. 1879; 1: 200-2, 214-5.

4. World Health Organization. Emergence of multi-drug resistant Neisseria gonorrhoeae - Threat of global rise in untreatable sexually transmitted infections. Disponível em: http://whqlibdoc.who.int/ hq/2011/WHO_RHR_11.14_eng.pdf.

5. Institute of Medicine. The Hidden Epidemic: Confronting Sexually Transmitted Diseases. Committee on Prevention and Control of Sexually Transmitted Diseases. Washington: National Academy Press; 1977.

6. Van der Heyden JH, Catchpole MA, Paget WJ, Stroobant A. Trends in gonorrhoea in nine Western European countries, 1991-6. Sex Trans Infect. 2000; 76:110-6.
7. Centers for Disease Control and Prevention. STD Surveillance 2006: Trends in Reportable Sexually Transmitted Diseases in the United States, National Surveillance Data for Chlamydia, Gonorrhea, and Syphilis. Disponível em: http://www.cdc.gov.

8. Swygard H, Sena AC, Leone P, Cohen MS. Gonorrhea. In: Klausner JD, Hook III EW, editors. Sexually Transmitted Diseases. Blacklick: McGraw-Hill; 2007. p. 99-107.

9. McCormack WM. Pelvic inflammatory disease. N Engl J Med. 1994; 330:115-9.

10. Cohen MS, Hoffman IF, Royce RA, Kazembe P, Dyer JR, Daly CC, et al. Reduction of concentration of HIV-1 in semen after treatment of urethritis: implications for prevention of sexual transmission of HIV-1. Lancet. 1997; 349:1868-73.

11. Santo I, Azevedo J, Cardoso J. A consulta de doenças sexualmente trasmissíveis do Centro de Saúde da Lapa - Caracterização da população utente e padrão epidemiológico das IST. Trab Soc Port Dermatol Venereol. 2007; 65:365-74.

12. Guidelines for the management of sexually transmitted infections. WHP headquarters. 2003. Disponível em: www.euro.who.int.

13. Cole MJ, Unemo M, Hoffmann S, Chisholm SA, Ison CA, Van de Laar MJ. The European gonococcal antimicrobial surveillance programme, 2009. Euro Surveill. 2011; 16 pii: 19995.

14. Martin I, Sawatzky P, Allen V, Hoang L, Lefebvre B, Mina $\mathrm{N}$, et al. Emergence and Characterization of Neisseria gonorrhoeae Isolates With Decreased Susceptibilities to Ceftriaxone and Cefixime in Canada: 2001-2010. Sex Transm Dis. 2012; 39:31623.

15. Bolan GA, Sparling PF, Wasserheit JN. The emerging threat of untreatable gonococcal infection. $\mathrm{N}$ Engl J Med. 2012; 366:485-7.

16. Deresinski S. Cephalosporin-resistant gonorrhea: it's just a matter of time. AIDS Alert. 201 1; 26:115-6. 